\title{
Comparación de Tres Fórmulas Dietéticas en Lactantes con Diarrea Aguda
}

\author{
Dra. Sylvia Ibáñez T.' ; Dr. Rubén Puentes R. '; Dr. Augusto Winter E. ${ }^{1-2}$; \\ Q.F. Juan Guajardo $S^{2}$; E. U. Aurota Jeraldo I. ' Nutr. Ana Jiménez A. **
}

\section{Comparisson of three Feeding Fórmulas for Infants with Acute Diarrhea}

In the three groups of infants admitted to a Pediatric Hospital in Santiago with acute gastroenteritis, and refer respectively with milk, curd, and curd - and - carrot formula, water, electroly te and nitrogen balance and clinical evolution were compared. With either formula, satisfactory nitrogen absortion rates and retention were obtained. There wexe no significant differences in intestinal passaje time, nor in the risk of the patients to develop torpid diarchea. Infants refed with curd - and - carrot formula had slightly higher water and electrolites loss by feces, but the differences were not statistically significant. In all three groups, fecal loss wete still prominent between days four 10 seven, and there were no increases in weight except those accounted by changes in body water content. It is concluded that nejther the presence of fjbers nor the use of lactose-free formulas will modify the clinical eyolution or the cuantity of feces in acute diathea of infancy.

(Key words: Acute diarthea. Refeeding. Milk formulas).

EI Sindrome diarreico agudo es frecuente en lactantes y niños pequeños de los estratos socio económicos bajos de la población, especialmente en los periodos estivales. Con frecuencia se requiere hospitalizar a estos pacientes para su hidratación y realimentación.

Diversos autores han investigado el importante deterioro de la absorción intestinal que original. 2.3 encontrandose ella francamente disminuida para nitrógeno, grasa, ejectrolitos $y$, algo menos, hidratos de carbono. Esta última, sin embargo, puede encontrarse selectivamente comprometida en casos de tolerancia secundaria a lactosa y sacarosa, siendo esta complicación frecuentemente la causa de evolución tórpida del sindrome $e^{4,5,6}$,

Estas alteraciones pueden persistir durante varias semanas ${ }^{2}$, con el consiguiente deterioro del estado nutricional del niño, ya muchas veces comprometido al ingresar al Hospital.

1. Servicio de Pediatría, Hospital Sóteto del Río. (S.S.M.S.O.),

2. Departamento de Pediatría. Facultad de Medjeina. Universidad Católica de Chile.

* Trabajo financiado en parte por la Dirección de Investigación de la Pontificia Universidad Católica de Chile. (Proyecto N-3280).
A pesar de las grandes pérdidas đe nutrientes y electrolitos por las deposiciones en la diarrea, la cantidad absoluta de nutrientes absorbidos y retenidos es proporcionalmente mayor cuando los aportes de estos en la dieta son más altos ${ }^{2}, 3$, g. Por ello, la persistencia de deposiciones alteradas no debe desanimar una adecuada realimentación durante el curso de la enfemedad.

Por otra parte, las fómulas lácteas comunes tienen el inconveniente potencial de agravar el problema en caso de intolerancia a disacáridos ${ }^{4}$. $5,6,12$, provocando diarrea osmótica.

Esto ha motivado a buscar fómulas que permitan realimentar con relativa seguridad al njno9, 10,11 con Síndrome diarreico agudo.

En nuestro Servicio se ha utilizado con este fin una fórmula pobre en lactosa, basada en quesilio de leche, con agregados de glucosa, aceite, y zanahoria cocida licuada. La zanahoria ha sido también frecuentemente agregada a fórmulas "licuadas" de pollo o carne y a mezclas para hiperalimentación enteral, por sus supuestas propiedades "astringentes" y para ayudar a mantener en suspensión los demás componentes de la fórmula.

Un estudio efectuado anteriormente en nuestro Servicio" demostró que la "Crema de Zanahoria" era clínicamente útil en la realimentación del Síndrome diarreico agudo, logrando 
más rápida normalización de las deposiciones que las fómulas lácteas, permitiendo un alta más precoz del niño.

En dicho estudio el método clínico no permitió determinar si la normalización acelerada de las deposiciones relacionada con la "Crema de Zanahoria". era real o sólo aparente, similar a la respuesta observada con harina de algarroba ${ }^{9} 0$ de garbanzo.

Pareció necesario, por lo tanto, efectuar un estudio con técnica de balance metabólico para determinar los efectos, tanto de la dieta sin lactosa, como de la fibra aportada en forma de zanahoria, en la realimentación del lactante con Diarrea Aguda.

\section{MATERIAL Y METODO}

Se incluyeron 31 lactantes de 3 meses a 1 año de edad, de sexo masculino ingresados al Servicio de Pediatría del Hospital Sótero del Río, por Síndrome Diarreico agudo con deshidratación. Se excluyó a los lactantes con desnutrición severa o con otra enfermedad concomitante (Bronconeumonia, Meningitis, Septicemia). Los pacientes fueron distribuidos aleatoriamente en 3 grupos de 11,10 y 10 niños respectivamente, recibiendo cada grupo realimentación diferente.

Durante las primeras 24 horas todos los niños recibieron aporte exclusivamente por vía paren. teral, según las normas de hidratación del Servicio. Desde el segundo dia recibieron la dieta asignada en volúmenes crecientes, hasta llegar a $150 \mathrm{cc} . \mathrm{x} \mathrm{kg}$. de peso al $4^{\circ}$ dia, manteniéndose igual volumen los dias siguientes. Los aportes hídricos y electrolíticos se completaron, según el estado de hidratación del niño, con soluciones endovenosas o con agua glucosada por vía oral.

El Grupo I se realimentó con leche de vaca en polvo (Purita ${ }^{\circledR}$ ) diluida en agua al 7,5\% con azúcar al $5 \%$ y Maltosa-dextrina (Nessucar (B)) al $5 \%$. El Grupo II recibió una fómula basada en quesillo de leche acidificada (Eledón ${ }^{\circledR}$ ) de $10 \mathrm{~g}$ . $100 \mathrm{ml}$ reconstituida en agua con glucosa al $7 \%$, aceite al $3 \%$ y un agregado de $20 \%$ de zanahoria cocida y licuada. El Grupo III recibió realimentación basada en quesillo de leche modificada (Eledón $\odot$ ). reconstituida con agua, gluco. sa al $7 \%$, aceite al $3 \%$, (sin zanahoria). A las dos últimas fórmulas se les agregó $0.5 \mathrm{~g}$. $\mathrm{x} 6 \mathrm{lt}$., de $\mathrm{NaCl}$ y de $\mathrm{KCl}$.

Las tres fómulas aportaron cantidades similares de calorias, proteínas, sodio y potasio. Estos tres últimos se midieron diariamente de una muestra de cada preparación (Tabla 1).

Finalizado el período de estudio, las fómulas de quesillo y crema de zanahoria fueron remplazadas progresivamente por Leche Purita ${ }^{(\mathbb{R}}$, antes del alta del niño.

Durante las primeras 24 hotas y entre los días 4 y 7 de hospitalización, los lactantes fueron manejados en camas metabólicas, para realizar balance nitrogenado e hidroelectrolítico.

En el primer balance la recolección de orina $y$ deposiciones se efectuó desde el ingreso hasta las 24 horas siguientes midiéndose, además, la velocidad de tránsito intestinal, medjante la administración oral de $0,05 \mathrm{~g}$. de rojo carmín.

Durante el segundo período, entre los días 4 y 7 se administró rojo carmín como indicador del inicio y término de la recolección de deposiciones $y$, además, para medir la velocidad del tránsito intestinal.

Se midió el volumen de orina en vaso gra. duado y se pesaron las deposiciones en balanza de precisión. Se pesó cuidadosamente cada riño antes y después de cada período de balance. Se hicjeron mediciones de $\mathrm{pH}$ y reacciones de Benedict en muestras de deposiciones obtenidas directamente, por sonda rectal, al ingresar el niño al estudio $y$ al finalizar el segundo período de balance.

Se midieron electrolitos (sodio y potasio) en el plasma, por fotometría de llama al inicio y térnino de cada balance; proteinemia (R. de Baiwat) al ingreso $y$ al finalizar el estudio; nitrógeno ureico ( $R$. de Berthelot) y creatinina (R. Yafhe) al ingreso, al comenzar y finalizar el segundo balance; Xilosa ( $R$. de Paracromoanilina), al finalizar el estudio, una hora después de adminis.

Tabla 1

Aportes por 100 cc de Preparación de las Fórmulas Usadas en el Estudio

\begin{tabular}{|c|c|c|c|c|c|c|}
\hline Grupos & $\frac{\text { Proteinas }}{(\mathrm{g})}$ & $\frac{\text { H. de C. }}{\text { (g) }}$ & $\frac{\text { Lípidos }}{\text { (g) }}$ & $\frac{\text { Calo. }}{\text { rías }}$ & $\frac{\mathrm{NA}}{(\mathrm{mEq})}$ & $\frac{K}{(m \overrightarrow{E q})}$ \\
\hline $\begin{array}{l}\text { Leche Purita } 7,5 \% \\
\text { Crema Zanahoria } \\
\text { Crema Quesillo }\end{array}$ & $\begin{array}{l}2,0 \\
2,2 \\
2,4\end{array}$ & $\begin{array}{r}12,6 \\
8,6 \\
7,0\end{array}$ & $\begin{array}{l}1,9 \\
3,3 \\
3,3\end{array}$ & $\begin{array}{l}75,5 \\
72,9 \\
67,3\end{array}$ & $\begin{array}{l}1,6 \\
1,8 \\
1,4\end{array}$ & $\begin{array}{l}2,5 \\
2,3 \\
1,4\end{array}$ \\
\hline
\end{tabular}


trar 5 g. de xilosa por sonda naso-gástrica y gases en sangre (I.L. 213 - 329) ál empezar cada balance.

En la orina se midió (en el volumen total obtenido en cada período de balance) el contenido de nitrógeno total (M Kjeldhal); los electrolitos (sodio y potasio) y la densidad.

En las deposiciones obtenidas de cada periodo de balance se midieron el nitrógeno tatal (M. Kjeldhal) y los electrolitos (sodio y potasio).

Se consideró que un lactante tenia diarrea de "evolución tórpida" o inhabitual cuando se constataba persistencia de la deshidratación más allá de las primeras 72 horas, pérdidas hídricas por deposiciones superiores en una o más desvia. ciones estándar respecto del promedio del grupo en el segundo periodo de balance, o recaida del síndrome diarreico en los días siguientes al témino del estudio.

El estado de la nutrición se evaluó en cada paciente según tablas del Centro Intemacional de la Infancia (M. Sempé).

\section{RESULTADOS}

La edad promedio del grupo fue 5,4 meses, oscilando entre 5 y 8 meses, La mayoría de los nirios eran eutróficos, con $37,5 \%$ de desnutridos leves, y $15,6 \%$ de desnutridos moderados (se excluyeron del estudio los lactantes con desnutrjción severa). La mayor parte pertenecía a los estratos socio-económicos bajo o medio bajo.

La duración de la enfermedad antes de la hospitalización, fue habitualmente menor de una sernana. En $87,5 \%$ de los casos hubo vómitos de alguna cuantia, y en $64,5 \%$ alzas térmicas febriles. Sólo $18,2 \%$ presentó, al ingreso, signos clínicos de deshidratación leve; el resto tenía signos graves y $6,1 \%$ ingresó con evidencia de pre-shock.
El tipo de deshidratación fue predominantemente isotónico. No se detectó hipernatremia en ningún niño, $30 \%$ tenia hiponatremia; hubo moderado grado de acidosis metabólica $(\mathrm{pH}$ promedio 7,27 , y exceso de base promedio de $-11,2 \mathrm{mEq} \times \mathrm{L})$. La proteinemia al ingresar estaba elevada, igual que el nitrógeno ureico plasmático $(18,8 \pm 11,8 \mathrm{mg} \%)$ y la creatinina plasmática $(0,78 \pm 0,28 \mathrm{mg} \%)$.

Al ingreso poco mas de la mitad de los lactantes $(n=18)$ tenía $\mathrm{pH}$ ácjdo en las deposi. ciones $y$ en 15 la reacción de Benedict era positiva, con distribución similar en los 3 grupos.

Durante el primer balance las cantidades ingeridas y las pérdidas por orina y deposiciones, de agua, nitrógeno, sodio y potasio fueron similares en los tres grupos. La velocidad del tránsito intestinal fue también similar en los tres $(5,3) \mathrm{h} \pm 3,1$ h) (Tabla 2).

En los electrolitos plasmáticos las concentraciones de sodio estaban en rango normal o bajo, $y$ el potasio normal. Ambos tendieron a mejorar con la hidratación.

El ascenso de peso, al mejorar el estado de hidratación fue de $49 \mathrm{~g} \pm 32$ (dos niños no tuvieron ascenso de peso significativo).

Durante el segundo período de balance los aportes orales de agua, sodio, potasio, nitrógeno, calorías, volumen de fórmula ingerja y electrolitos por via parenteral, en los casos que los requirieron, fueron similares en los tres grupos (Tabla 3).

Las pérdidas de agua por orina fueron igualmente bajas en los 3 grupos $(36 \pm 16 \mathrm{cc}$. $\mathrm{x} \mathrm{Kg}$. de peso). No se constató poliuria en ningún niño durante el segundo período de balance.

Los volúmenes de deposiciones fueron algo mayores en el grupo realimentado con Crema de Zanahoria, especialmente en los nifios que evolucionaron en forma tórpida. Sin embargo esta

Tabla 2

Balance de Hidroelectrolítico y Nitrogenado de Primeras 24 Horas de Hospitalización en Lactantes con S.D.A.

\begin{tabular}{|c|c|c|c|c|c|c|c|c|c|}
\hline \multirow{3}{*}{$\frac{\text { Elementos }}{\text { Agua (cc. } x \mathrm{Kg}-\mathrm{p} \text { ) }}$} & \multirow{2}{*}{\multicolumn{3}{|c|}{ Ingresos }} & \multicolumn{6}{|c|}{ Egresos } \\
\hline & & & & \multicolumn{3}{|l|}{ Orina } & \multicolumn{3}{|c|}{ Deposiciones } \\
\hline & 198,0 & \pm & 43,0 & 54,0 & \pm & 30,0 & 40,0 & \pm & 22,0 \\
\hline \multicolumn{10}{|l|}{ Nitrógeno } \\
\hline (mg. $x \mathrm{Kg}-\mathrm{p})$ & 0,0 & & & 158,0 & \pm & 95,8 & 23,5 & \pm & 57,0 \\
\hline $\mathrm{Na}(\mathrm{mEq} \times \mathrm{Kg}-\mathrm{p})$ & 14,0 & \pm & 3,9 & 1,6 & \pm & 1,9 & 2,3 & \pm & 1,9 \\
\hline $\mathrm{K}(\mathrm{mEq} \cdot \mathbf{k g}-\mathrm{p})$ & 2,3 & \pm & 0,9 & 0,6 & \pm & 0,6 & 1,3 & \pm & 0,8 \\
\hline \multicolumn{10}{|l|}{ Calorías } \\
\hline (K. cal $\times \mathrm{kg}-\mathrm{p}$ ) & 59,4 & \pm & 12,9 & & & & & & \\
\hline
\end{tabular}


diferencia no fue significativa en comparación a los otros dos grupos. Esta tendencia a mayores pérdidas en el grupo realimentado con Crema de Zanahoria se observó tambièn en relación con el nitrógeno, sodio y potasio, aunque tampoco las diferencias fueron significativas. (Tabla 4.).

Llamó la atención en el segundo periodo que las deposiciones persistieran de aspecto liquido y con volumen promedio de $41 \mathrm{cc}$. $\times \mathrm{Kg}$ de peso, en forma similar a las pérdidas observadas en las primeras 24 horas. La velocidad promedio del tránsito intestinal al término del segundo período fue semejante en los tres grupos, algo menor que el ingreso, aunque no significativamente, $y$ siem- pre acelerada en relación con la velocidad del tránsito normal. (Tabla 5 ).

La absorción de nitrógeno fue de $71 \% \pm 16$ en todo el grupo, con $71 \% \pm 11$ para la Leche Purita, $64 \% \pm 23$ para la Crema de Zanahoria $y$ $76,7 \% \pm 9.3$ para la fórmula de quesillo, no siendo estas diferencias significativas. En el grupo con Crema de Zanahorias el promedio más bajo se debió a un caso con absorción de $12 \%$ teniendo el resto del grupo absorción promedio de $70 \% \pm 16$. La retención nitrogenada fue positiva en 30 de los 31 casos, con un promedio de $45 \% \pm 12$. Fue negativa en un solo caso (el mismo que tuvo una absorción de $12 \%$ ). La canti-

Tabla 3

Aportes Recibidos por los Lactantes con S.D.Al en e1 $2^{\circ}$ Período de Balance $\left(4^{\circ}\right.$ a $7^{\circ}$ Días)

\begin{tabular}{|c|c|c|c|c|c|c|}
\hline $\begin{array}{l}\text { Fórmula recibida } \\
\text { por cada grupo }\end{array}$ & $\begin{array}{l}\text { Vohumen } \\
\text { Fórmula } \\
\text { (ce } x \mathrm{~kg} x \\
\text { dia) }\end{array}$ & $\begin{array}{l}\text { Proteínas } \\
\text { (g. x kg x } \\
\text { día) }\end{array}$ & $\begin{array}{l}\text { Calorías } \\
\text { (K cal. } x \\
\text { día) }\end{array}$ & $\begin{array}{c}\text { Total } \\
\text { aporte } \\
\text { (cc x Kg. } \\
\text { dia) }\end{array}$ & $\begin{array}{c}\mathrm{Na} \\
(\mathrm{mEq} \times \\
\mathrm{K} \mathrm{B}^{\mathrm{x}} \times \mathrm{día}\end{array}$ & $\underset{\text { dia })}{K} \underset{\mathrm{mEq} \times \mathrm{Kg} \times}{\mathrm{K}}$ \\
\hline Leche Purita $7.5 \%$ & 136 & 2,6 & 107 & 154 & 2,8 & 3,4 \\
\hline Crema Zanahoria & 135 & 2,8 & 100 & 166 & 4,8 & 4,0 \\
\hline Crema Quésillo & 143 & 3,3 & 104 & 168 & 3,5 & 2,4 \\
\hline
\end{tabular}

Tabla 4

Pérdidas por Deposiciones Diarias y Balance Nitrogenado entre el $4^{\circ}$ y $7^{\circ}$ Días de Evolución, en 31 Lactantes con S.D.A.

\begin{tabular}{|c|c|c|c|c|c|c|}
\hline \multirow[b]{2}{*}{ Grupos } & \multirow{2}{*}{$\begin{array}{l}\text { Volumen } \\
\text { Depos. } \\
(\operatorname{cc~} x \mathrm{Kg})\end{array}$} & \multicolumn{3}{|c|}{ Nitrógeno } & \multirow{2}{*}{$\begin{array}{c}\mathrm{Na} \\
(\mathrm{mEq} \\
\mathrm{x} \mathrm{Kg.})\end{array}$} & \multirow{2}{*}{$\begin{array}{c}K \\
(\mathrm{mEq} \\
\times \mathrm{Kg} .)\end{array}$} \\
\hline & & $\begin{array}{l}\text { Pérdida } x \text { dep. } \\
\text { (mg. } \times \text { Kg.) }\end{array}$ & $\begin{array}{c}\% \\
\text { Retención } \mathrm{N}\end{array}$ & Absorción & & \\
\hline Leche Purita 7,5\% & $39,5 \pm 15,5$ & $116,8 \pm 44,4$ & $44,8 \pm 12,6$ & $71,3 \pm 10,7$ & $1,5 \pm 0,7$ & $1,2 \pm 0,5$ \\
\hline Crema Zanahoria & $49,3 \pm 25,3$ & $139,5 \pm 90,0$ & $45,6 \pm 12,0$ & $64,0 \pm 23,0$ & $2,0 \pm 1,5$ & $1,5 \pm 0,8$ \\
\hline Crema Quesillo & $38,8 \pm 22,8$ & $119,1 \pm 45,0$ & $46,9 \pm 12,3$ & $76,7 \pm 9,3$ & $1,2 \pm 1,2$ & $0,7 \pm 0,6$ \\
\hline
\end{tabular}

Tabla 5

Repercusión del Cuadro Diarreico en la Función Digestiva durante el Segundo Período de Balance

\begin{tabular}{|c|c|c|c|c|}
\hline Grupos & $\begin{array}{c}\mathrm{pH} \text { en } \\
\text { Deposiciones } \\
<6\left(4^{\circ} \text { día }\right)\end{array}$ & $\begin{array}{l}\text { Benedict } \\
\text { Positivo } \\
\left(4^{\circ} \text { dia) }\right.\end{array}$ & $\begin{array}{l}\text { Velocidad } \\
\text { Tránsito } \\
\text { [ntestinal Hrs. } \\
\text { (70 día) }\end{array}$ & $\begin{array}{c}\text { Test-Xilosa } \\
\text { mg. \% } \\
\left(7^{0} \text { día }\right)\end{array}$ \\
\hline $\begin{array}{l}\text { Leche Purita } 7,5 \% \\
\text { Crema Zanahoria } \\
\text { Crema Quesillo }\end{array}$ & $\begin{array}{l}18,2 \% \\
60,0 \% \\
40,0 \%\end{array}$ & $\begin{array}{l}9 \% \\
10 \% \\
10 \%\end{array}$ & $\begin{array}{l}6,7 \pm 3,0 \\
7,5 \pm 2,5 \\
8,6 \pm 5,7\end{array}$ & $\begin{array}{l}32,7 \pm 17,5 \\
26,0 \pm 15,0 \\
26,9 \pm 11,6\end{array}$ \\
\hline
\end{tabular}


dad absoluta de nitrógeno retenido fue de 194 $\mathrm{mg} x \mathrm{~kg}$ de peso con leche; $212 \mathrm{mg} \mathrm{x}$ kg de peso con crema de zanahoria y $256 \mathrm{mg} x \mathrm{~kg}$ de peso con quesillo, encontrándose la mayoría de los casos entre un rango de 100 y $300 \mathrm{mg}$. de Nitrógeno $\mathrm{x} \mathrm{kg}$ de peso, lo cual representa una retención diaria de 0.6 a $1.9 \mathrm{~g}$ de proteína $x \mathrm{~kg}$ de peso.

El nitrógeno ureico plasmático tendió a normalizarse en relación al ingreso, con leve tendencia a aumentar entre el inicio y término del segundo balance $y$ sin diferencias significativas entre los tres grupos. Lo mismo ocurrió con el promedio de creatinina plasmática. Al 70 dia el valor promedio de proteinemia total fue de 6.63 $\mathrm{mg} \% \pm 0.9$. En los tres grupos hubo niños con proteinemias bajo lo normal (En 8 los valores eran inferiores a $6.3 \mathrm{mg} . \%$ ).

La concentración promedio de xilosa plasmatica fue de $28.6 \pm 15,3 \mathrm{mg}$. \% sin diferencias significativas entre los tres grupos; casi la mitad de los niños tuvo en las pruebas de xilosa valores inferiores a los normales.

Natremia y Kalemia mostraron al séptimo día discreta tendencia a aumentar, observándose $19 \%$ de niños con hiponatremia y ninguno con hipernatremia.

Al comienzo del segundo balance, dos de once niños realimentados con leche tuvieron $\mathrm{pH}$ de deposiciones inferior a 6 y otro reacción de Benedict positiva. Del grupo realimentado con Crema de Zanahoria, seis tuvieron $\mathrm{pH}$ inferior a 6 y uno Benedict positivo. En los niños realimentados con quesillo, cuatro tuvieron $\mathrm{pH}$ inferior a 6 y ninguno Benedict positivo.

En el grupo realimentado con leche, dos niños sufrieron recaída del sindrome diarreico y uno evolucionó como diarrea tórpida, demostrándose sólo en éste intolerancia por la lactosa.

Tres niffos realimentados con Crema de Zanahoria, $y$ dos niños realimentados con quesillo, evolucionaron también con diarrea tórpida.

Hubo otros dos niños (uno realimentado con leche y otro con quesillo) con grandes pérdjdas por deposiciones en el segundo período de balance, a pesar de lo cual no presentaron deshidratación después de las primeras 48 horas y las heces eran formadas al término del estudio.

El aumento de peso durante todo el periodo de estudio fue, en los 3 grupos, prácticamente igual al alza experimentada en las primeras 24 horas de hidratación parenteral, por lo tanto, atribuíbles sólo a cambios de hidratación.

\section{DISCUSION}

El retardo y la interferencia con la absorción de nutrientes por las fibras de la dieta están ampliamente demostrados. En el individuo nor. mal una dieta rica en fibras produce deposiciones de mayor volumen y contenido de agua, electrolito, minerales, nitrógeno y grasas que una dieta pobre en fibra $18,20,21,22,23$, como también retraso y aplanamiento en la curva de absorción de glucosa.

Este efecto se observa especialmente con polisacáridos no absorbibles de cadena larga (lignina, celulosa); pero tambièn se ha observado mayor arrastre de nutrientes con algunos polisa. cáridos no absorbibles de cadena corta, como la pectina $^{7}, 21$.

La zanahoria cocida según Southgate ${ }^{19}$, posee $1,5 \%$ de celulosa y $2.2 \%$ de polisacáridos de cadena corta. Según esta información un volumen de 135 cc. $x \mathrm{Kg}$. peso de "Crema de Zanahoria", cantidad que corresponde a la aportada en este trabajo a uno de los grupos de estudio, suministra aproximadamente $1 \mathrm{~g}$ de fibra por $\mathrm{kg}$ de peso. No hubo pérdidas de agua, electrolitos o nitrógeno significativamente mayores en el grupo que recibió crema de zanahoria,

A pesar de que se trataba de lactantes con alteraciones evidentes de la absorción, como lo ilustran las pruebas de vilosa alteradas al $7^{\circ}$ dia en un número importante de niños, no se constató tampoco cambios en la velocidad del tránsito intestinal en los pacientes realimentados con zanahoria, siendo la incidencia de diarrea de evolución tótpida parecida en los tres grupos de riños. Estos resultados contrastan con la evolu. ción más favorable de los pacientes con SDA realimentados con crema de zanahoria vs leche, registrada en un trabajo previo del mismo servicio $^{11}$ basado en la evolución clínica del aspecto de las deposiciones.

Esta discordancia entre la apreciación clínica y el balance metabólico en niños con Sindrome diarreico agudo fue también observada por Duffau y colaboradores en un estudio comparativo entre realimentación con harina de garbanzo (GL) y leche, en que la mejoría más precoz de las deposiciones con GL fue sólo aparente. Algo semejante se observó en un estudio hecho para evaluar el uso de harina de algarroba, en el cual, a pesar del aspecto normal de las deposiciones, se detectaron pérdidas aumentadas de nitrógeno $\mathrm{y}$ de electrolitos al agregar el producto a una fórmula láctea ${ }^{9}$.

En este estudio no se observaron cambios en el curso de la diarrea aguda el usar fómulas sin lactosa en la realimentación inicial.

En el grupo realimentado con leche, a pesar de que cuatro niños tenian $\mathrm{pH}$ de deposiciones inferior a 6 y tres Benedict positivo al ingresar, sólo en dos persistía pH ácido, y en uno Benedict positivo al término del estudio, siendo este últirno el único con pérdidas importantes por las 


\section{deposiciones.}

La persistencia de pérdidas aún importantes al séptimo día en gran parte de los lactantes, asi como la falta frecuente de correlación entre la evaluación clinica de las deposiciones y las pérdidas comprobadas en la cama metabólica, son hechos que vale la pena destacar, y que han sido observados también por otros autores $2,15 \mathrm{y}$ subrayan la utilidad del balance metabólico cuando se sospecha una evolución tórpida.

A pesar de persistir pérdidas importantes en el segundo período del estudio, el balance nitrogenado fue positivo en la mayoria de los casos con cualquiera de las fómulas, lo que no se acompañó de aumento concomitante del peso, posiblemente por estar los pacientes en etapa de reparación de tejidos.

\section{RESUMEN}

Se comparan los balances de agua, sales y nitrógeno y la evolución clínica, en 3 grupos de lactantes con Síndrome diarreico agudo, uno de los cuales se realimentó con leche, y los otros dos con quesillo de Eledón reconstituido, con y sin agregado de zanahoria respectivamente. Con las 3 fórmulas se obtuvo absorción y retención nitrogenada satisfactorias. No hubo diferencias significativas en la velocidad del trânsito intestinal al $7^{\circ}$ día, ni en la incidencia de evolución tórpida del cuadro diarreico. El grupo que recibió zanahoria en la dieta presentó pérdidas hidroelectrolíticas por deposiciones algo mayores, aunque no estadisticamente significativas. Destacó la persistencia de pérdidas importantes por deposiciones entre el $4^{\circ}$ y $7^{\circ}$ dias, $y$ la ausencia de ganancia ponderal (excepto por cambios de hidratación) en los tres grupos, no obstante lo cual se obtuvo balance nitrogenado positivo en la mayoría de los lactantes. Se concluye que la presencia de fibra en la dieta, o el uso de fómulas sin lactosa, no modifican ostensiblemente la evolución ni la magnitud de las perdidas por deposiciones en el Sínd rome diarreico agudo del lactante.

\section{REFERENCIAS}

1. Hugo de Rivera $C$, Rodríguez H., Torres Piñedo R.: Estudios en mecanismos de mala absorción de azúcares en diarrea infecciosa infantil. Am. J. Clin. Nutr. 25: 1248, 1972.

2. Ayesho $M$ : Efecto de la diarrea en absorción de macronutrientes en el estado agudo y después de la recuperación. Bellagio Conference Center - Beilagio, Italy, Mayo 11-15, 1981.
3. Chung A.: The effect of oral feeding at different levels on the absortion of food stuff in infantile djarrhea. J. Pedíatr. 33: 1, 1948.

4. Emilfork, $M$. Duffou, $G$.: Síndrome diarreico de evolución tórpida del lactante. Intolerancia secundaria a hidratos de carbono. Metódica de estudio. Pectiatría (Santiago), 17: 107, 1974.

5. Donús $O$, Urbina $M$. Soriano $H$. y Cols: Intolerancia a la lactosa en el síndrome diarreico agudo del lactante. Pediatría (Santiago), 13: 51, 1970.

6. Duffau G., Emilfork M. Macaya Y. y Cols.: Síndrome diarreico agudo de evolución tórpída del lactante. Intolerancia secundaria a sacarosa. Pediatría (Santiago), 17: 103, 1974.

7. Jorpa $S_{*}$ Morrner Y.: Estudio metabólico en lactantes desnutridos alimentados con harina de garbanzos. Rov. Chil. Pediatr. 44: 395, 1973.

8. Chung $A$.: Efecto de la alimentación precoz versus ayuno precoz en el Síndrome diarreico agudo. 1. Pecliatr. 14: 22.1948.

9. Martner $Y$., Jarpa $S$., Biancani $F_{\text {.: }}$ Estudio comparativo entre leche semidescremada y otra similar con agregado de harina de algarroba. Rev. Chil. Pediatr. 44: 407, 1973.

10. Garcia $S$, Harum A.: Empleo de harina de garbanzo en el tratamiento del Síndrome diarreico agudo del lactante. Rev, Chil Pedjatr. 46: 319, 1975.

11. Ibóñez $S$, León L., Funez F., Puentes R., Gallo A.: Realimentación con crema de zanahoria de lactantes con Síndrome diarreico agudo. Rev. Chil Pediatr. 50: $S, 1979$.

12. Guiraldes $E$.: Intelerancia a los hidratos de carbono en la infancia. Rev. Chil. Pediatr. 46: 163, 1975.

13. Macaya J., García N., Lanzas R.: Organización de un Servicio para investigaciones metabólicas. Pedia. tria (Santiago), 15; 479, 1972.

14. Macayo $Y .$, Duffau G., Soriano $H$. Sindrome diarreico agudo con deshidratación del lactante. Evolución tótpida. Metódica de estudio. Pediatría (Santiago), 15: 488, 1972.

15 Macaya $Y .$, Duffal $G$., Soriano $H$. y Col: Técnica de balance en el Sindrome diarreico del lactante. Bol. Med. Hosp. Inf. Mex, 36: 541, 1979.

16. Pax $N$, Bernier $L$, Duffau $G$, y Cols: Formula de garbanzo en el tratamiento dietético del Síndrome diarreico agudo con deshidratación del lactante. Pediatría (Santiago), 17: 17, 1974.

17. Emilfork $M$, Duffou $G$, Macaya J.: Síndrome diarreico agudo del lactante. "Estudio con dos fórmulas dietéticas". Pediatría (Santiago), 17: 75, 1974.

18. Floch NH., Fuch HM.: Modificación del contenido fecal por aumento de la ingesta de fibra. Amer. $\mathrm{J}$. Clin. Nutr. 185: 1978.

19. Southgote D.: Fiebre dietarie. Amer, J. Clin. Nutr. 30: $303,1976$.

20. Cumings Y.H.: Changes in fecal composition and colonic function due to cereal fiber. Amer. J, Clin. Nutr. 29: 1461, 1976.

21. Cumings Y.H.: Nutritional implications of dietary fíber. Amer. J. Clin. Nutr. 31: 521, 1978.

22. Espinaza $J$, Krause $S .+$ Araya $M$., Brunser $O$.: Influencia de la fibra dietaria en excreción fecal de grasas. Rev, Chil. Nutr. 1: 113, 1981.

23. Kelsoy Y.L.: Effect of diet fiber on bowel function and trace mineral balance of human subjects. Cereal Chemistry. $58: 2,1981$. 